\title{
Detection of phase disturbances of free core nutation of the Earth and their concurrence with geomagnetic jerks
}

\author{
Toshimichi Shirai ${ }^{1}$, Toshio Fukushima ${ }^{2}$, and Zinovy Malkin ${ }^{3}$ \\ ${ }^{1}$ Goldman Sachs (Japan) Ltd., Minato-ku, Tokyo 106-6147, Japan \\ ${ }^{2}$ National Astronomical Observatory of Japan, Mitaka, Tokyo 181-8588, Japan \\ ${ }^{3}$ Institute of Applied Astronomy of the Russian Academy of Sciences, St. Peterburg 191187, Russia
}

(Received July 27, 2004; Revised January 17, 2005; Accepted January 29, 2005)

\begin{abstract}
We detected phase disturbances of the Free Core Nutation (FCN) of the Earth directly from the analysis of the Very Long Baseline Interferometer (VLBI) observation of the Earth rotation for the period 1984-2003 by applying the Weighted Wavelet Z-Transform (WWZ) method and the Short-time Periodogram with the Gabor function (SPG) method. During the period, the FCN had two significant phase disturbances in 1992 and 1998. These epochs coincide with the reported occurrence of geomagnetic jerks.
\end{abstract}

Key words: Free Core Nutation, reference frame, geomagnetic jerk.

\section{Introduction}

The Earth rotation possesses a free nutational mode rotating clockwise (retrograde) with the period about 430 sidereal days as viewed from the inertial frame. This mode, named as the Free Core Nutation (FCN), appears since the Earth has a rotating and elliptical fluid core (Toomre, 1974). The FCN influences the Earth rotation in two different ways; (1) modification of forced nutation terms through an indirect response as a non-rigid body, and (2) direct appearance of free oscillation modes. Investigation of the FCN is important in order not only to improve theoretical modeling of the nutations but also to understand nature of the Earth's interior. For instance, the period of the FCN is closely related to the dynamical ellipticity of the liquid outer core while the quality factor (Q-value) of the FCN is related to the viscosity of the outer (liquid) core and the core-mantle coupling (Sasao et al., 1980; Getino and Ferrandiz, 2000).

The time variation of the FCN amplitude has been well studied from the direct effect in the VLBI data (e.g. Shirai and Fukushima, 2001b; Dehant et al., 2003). A recent research also suggests that the atmosphere could have sufficient power to excite the FCN amplitude observed (Dehant et al., 2003) and huge earthquakes could cause impulsive changes of the FCN amplitude (Shirai and Fukushima, 2001b). In contrast, most of recent determinations of the FCN period are based on the estimation of the indirect effect using VLBI nutation of the Earth rotation (e.g. Shirai and Fukushima, 2001a) or monitoring of the tidal variation of the gravity (e.g. Sato et al., 1994). Usually these works assumed the FCN period as a constant value. Therefore the time variation of the FCN period has been unavailable. Exceptions are Roosbeek et al. (1999) and Hinderer

Copy right(c) The Society of Geomagnetism and Earth, Planetary and Space Sciences (SGEPSS); The Seismological Society of Japan; The Volcanological Society of Japan; The Geodetic Society of Japan; The Japanese Society for Planetary Sciences; TERRAPUB et al. (2000), who analyzed the time variation of the FCN period from the indirect effect in the VLBI data. They concluded that the FCN period has been stable with a precision of three sidereal days. One disadvantage of the determinations based on the indirect effects is that the time variation of the FCN phase is ignored. In general, the analysis of the time variation of the FCN frequency consists of two factors; that in period and that in phase. However the time variation of the FCN phase has not been studied yet. By directly applying the Wavelet Weighted Z-Transform (WWZ) method and the Short-time Periodogram with Gabor function (SPG) method to the VLBI nutation data, we try to reveal the time variation of the FCN phase and discuss its origin.

\section{Method of Analysis}

The methods we deployed in the analysis are the WWZ method and the SPG method. Each method has its own advantages and disadvantages. The WWZ method was initially developed for the period analysis of variable stars by Foster (1996), where time series of data are unevenly sampled. One of its merits is that the WWZ traces the time variation of both the amplitude and period at the same time. On the other hands, this method cannot treat complex-valued data, or vector data in general, and does not take care of the data with different weights. The WWZ method does not cover all the requirements of FCN analysis, where the data are usually expressed in a complex value (Shirai and Fukushima, 2001a) as

$$
\zeta_{\mathrm{FCN}}=\Delta \psi_{\mathrm{FCN}} \sin \epsilon_{0}+i \Delta \epsilon_{\mathrm{FCN}} .
$$

Here $\Delta \psi_{\mathrm{FCN}}$ is the FCN in longitude, $\Delta \epsilon_{\mathrm{FCN}}$ is that in obliquity, and $\epsilon_{0}$ is the obliquity of ecliptic at J2000.0. Note $\Delta \psi_{\mathrm{FCN}}$ and $\Delta \epsilon_{\mathrm{FCN}}$ are projections of axis motion of the Earth rotation to two rectangular coordinates viewed from the space. Also the VLBI data set contains the information on the weights of observed values of $\zeta_{\mathrm{FCN}}$. Then it is im- 


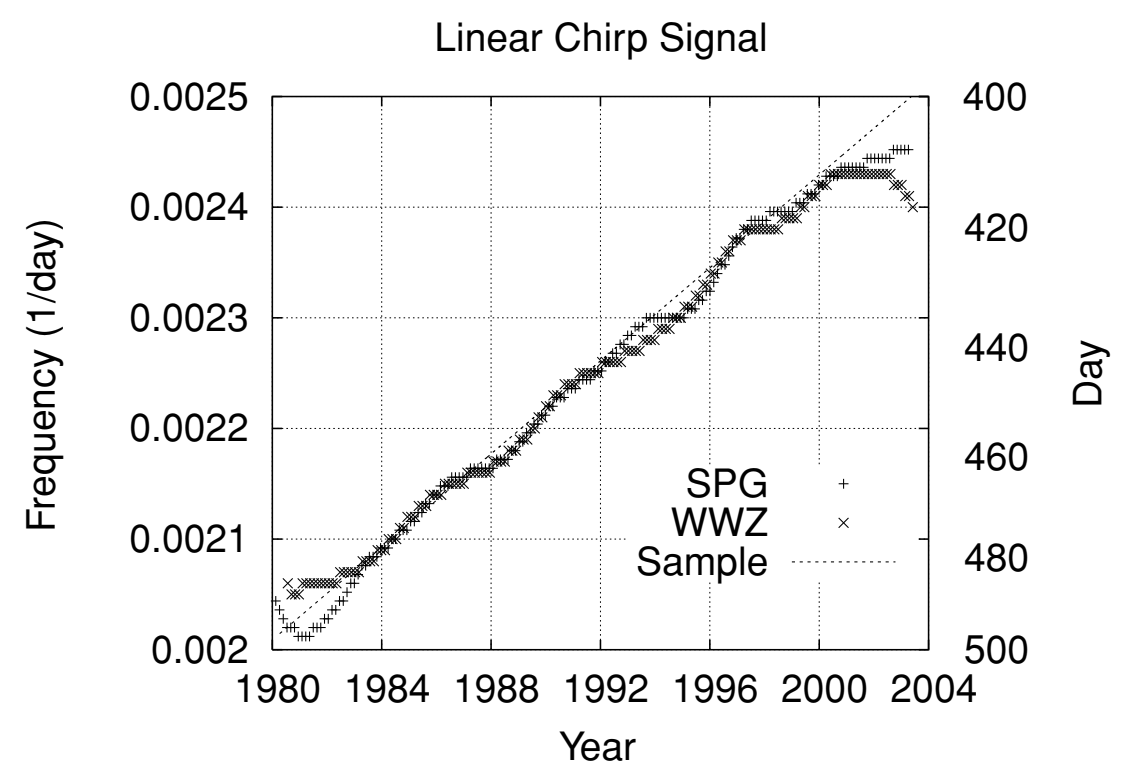

Fig. 1. Both the WWZ method and the SPG method precisely traced the chirp signal. The RMS for the SPG method and the WWZ method are 3.4 day and 4.5 day, respectively.

portant to consider them for the data before 1990, when the observation was not so precise.

The SPG method was developed by ourselves to handle unevenly sampled and complex-valued (i.e., in twodimension) time series with weights. Let us consider some sinusoidal signals embedded in unevenly sampled and complex observation time series as

$$
z_{j}=x_{j}+i y_{j}
$$

with different observational errors, respectively $\delta x_{j}$ and $\delta y_{j}$. Here the subscript $j$ on values corresponds to those at $t_{j}$. First, we assume that the data are associated with the standard weights

$$
w_{j}=\frac{1}{\left(\delta x_{j}\right)^{2}+\left(\delta y_{j}\right)^{2}} .
$$

In addition, we adopted the Gabor function based on the Gaussian function as an optimized window function for the time-frequency analysis (Gabor, 1946).

$$
G_{b}^{\alpha} \equiv g_{\alpha}(t-b)
$$

where

$$
g_{\alpha}(\tau)=\frac{1}{2 \sqrt{\pi \alpha}} e^{-\frac{\tau^{2}}{4 \alpha}}
$$

Here $\alpha$ is a trade-off parameter between time-resolution and frequency-resolution, and $b$ is a center epoch for the time shift. A larger $\alpha$ corresponds to a better frequency resolution. The time-frequency analysis would be achieved by shifting $b$ discretely as $b_{k}=b_{0}+\Delta_{b} k$ where $\Delta_{b}=$ $b_{1}-b_{0}$. To obtain periodogram at the specific epoch $b_{k}$, a least square fitting of sinusoidal curve to the windowed data $w G_{b_{k}}^{\alpha} z$. Here $\omega_{l}$ is discretely divided frequency as $\omega_{l}=\omega_{0}+\Delta_{\omega} l$ where $\Delta_{\omega}=\omega_{1}-\omega_{0}$. Then periodogram $P_{b}^{\alpha}\left(\omega_{l}\right)$ at specific epoch $b_{k}$ is evaluated as

$$
P_{b_{k}}^{\alpha}\left(\omega_{l}\right)=\frac{\rho\left(\omega_{l}\right)}{\sigma\left(\omega_{l}\right)}
$$

where

$$
\rho\left(\omega_{l}\right)=\sum_{j} G_{b_{k}}^{\alpha} w_{j} z_{j} e^{-i \omega_{l} t_{j}}, \sigma\left(\omega_{l}\right)=\sum_{j} w_{j} .
$$

To trace period changes during observation period, we just pick up the frequency of the maximum amplitudes from the periodogram at each epochs. One disadvantage of this method is that the accurate time variation of the amplitude is unavailable. Note that the estimated frequency variation would include not only the time variation of the FCN period but also that of its phase.

We performed a simple simulation with artificial test data to compare effectiveness of these methods. We created a test data set of unevenly sampled chirp signals whose frequency is slowly changing as a linear function of time ( $\left.f=f_{0}+f_{1} t\right)$. The timings of sampling were set the same as those of the actual VLBI data for the FCN analysis. Of course, we added Gaussian noise of small amplitude. Figure 1 shows that both the WWZ and the SPG methods precisely traced the chirp signal. We confirmed that the socalled edge effects, which has already been reported (Foster, 1996; Malkin and Terentev, 2003), are observed around the first and last 2 to 3 years. From the viewpoint of the RMS after fitting, we conclude that the SPG method is superior to the WWZ method. In fact, the post-fit RMS for the SPG method is 3.4 day, which is smaller than 4.5 day for the WWZ method. In any sense, the RMS for the both methods are small enough to trace the time variation of the FCN frequency of the Earth.

\section{Analysis of VLBI Data}

We used the VLBI nutation data complied by the U.S. Naval Observatory for the period MJD 44089.994 to 52779.206. Figure 2 shows residuals after subtraction of a standard model of the forced nutation, the IAU2000A (Mathews et al., 2002). Figure 2 clearly illustrates the existence of the FCN. Note that nutation data before 1984 


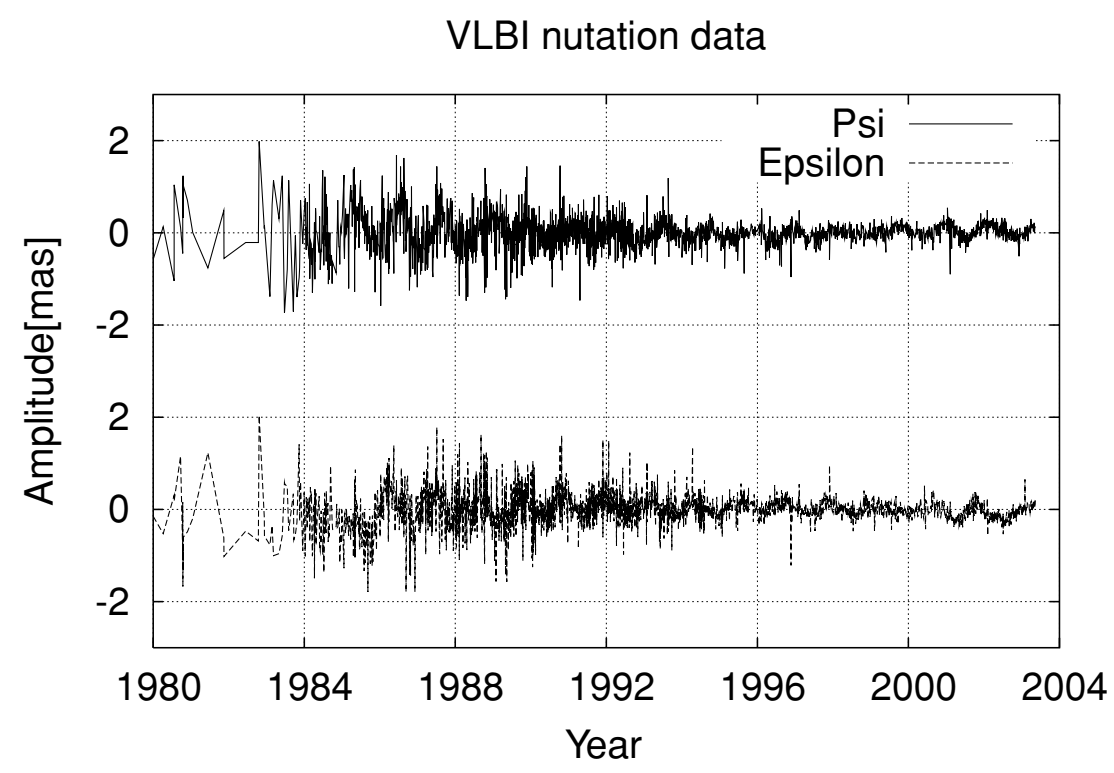

Fig. 2. Residuals after subtraction of a standard model of the forced nutation, the IAU2000A. Note that nutation data before 1984 is too noisy and has few data point.

shall not be used for our later analysis since they are too noisy and have too few data points. In the case of the WWZ method, we applied it separately to $\Delta \psi$ and $\Delta \epsilon$ without weights. This is because the WWZ method cannot handle the vector data with variable weights as explained.

As we mentioned before, the FCN period is closely related to the dynamical ellipticity of the outer liquid core, which is supposed to be constant in a short timescale. Additionally if FCN period significantly changes during the observation, the magnitude of the forced nutation itself must be also affected through the resonant effect. However Figure 2 shows no signs of such effects. Therefore it is quite natural to treat the FCN period as a constant and assume that the time variation of the FCN frequency mainly comes from that of the FCN phase. We calculate the time variation of the FCN phase from that of the FCN frequency by fixing the FCN period to a constant determined from the indirect effect, namely 430 sidereal days (Shirai and Fukushima, 2001a). We write the deviation of the phase from a linear function of time as

$$
\Delta \phi(t)=\phi(t)-2 \pi v_{\mathrm{FCN}}\left(t-t_{0}\right)
$$

where

$$
\phi(t)=\int_{t_{0}}^{t} 2 \pi v(t) d t .
$$

Here $v(t)$ is the observed time variation of the FCN frequency revealed by the SPG method or the WWZ method, $\nu_{\mathrm{FCN}}$ is the constant FCN frequency, and $\Delta \phi(t)$ is the time variation of the FCN phase.

Figure 3 shows the large time variation of the FCN phase. The figure indicates that there are two sudden trend changes of the FCN phase in 1992 and 1998. Before and after those sudden changes in trend, the time variation of the FCN phase is approximated by a linear function or ERF function which is the error function encountered in integrating the normal distribution. The result estimated by the SPG method is slightly different from that by the WWZ method, however, they are practically the same. We suppose that the result estimated by the SPG method is more accurate since the SPG method considers weights while the WWZ does not.

The first concern is whether this result is an artifact or not. The results of the numerical tests strongly suggests that this large time variation is not an artifact. The consistency between the results estimated by the two different approaches also supports that our result is not an artifact. Secondly we need to consider possibility of contaminations caused by the excitations of the FCN since neither the WWZ method nor the SPG method take account of those effects explicitly. One good candidate of the FCN excitation mechanism is atmosphere. It is hard to estimate the atmospheric effects on our result since the atmospheric model in diurnal time scale is not well known. A recent research suggests that the period of maximum atmospheric excitation varies with time, and it was closest to the FCN period around 1987 (Dehant et al., 2003). If the atmospheric excitation is mainly responsible for these period and/or phase variation, the maximum time variation should have happened around 1987. However Figures 2 and 3 show no signs of such effects. The other candidate of the FCN excitation mechanism is huge earthquake (Shirai and Fukushima, 2001b). It is also hard to estimate huge earthquakes effects on our result because the theoretical model is unavailable. However it is clear that earthquake signatures are invisible in Fig. 3. Therefore we conclude that their excitation effects on our result is insignificant.

\section{Discussion}

The FCN phase does not change without changes in its forcing. Let us discuss the geophysical mechanism of the FCN phase variation. We note that ten phase disturbances have been observed in the Chandler Wobble (CW) 18901997 with durations between 1 to several years (Gibert et 


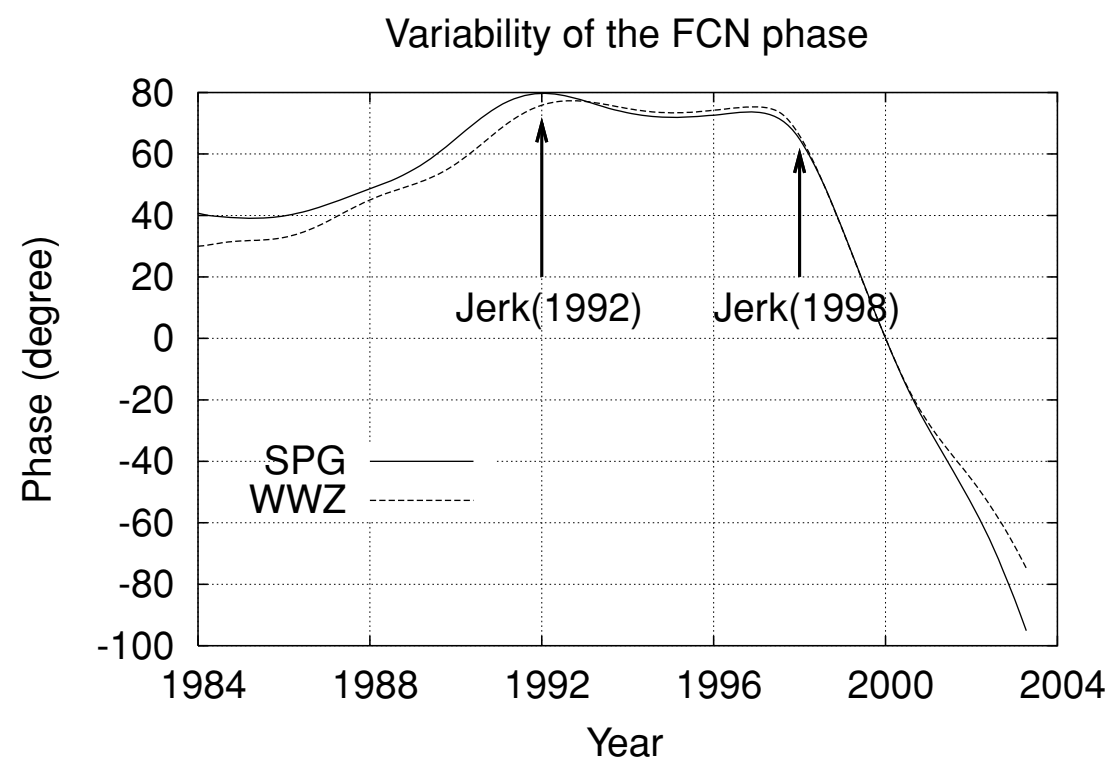

Fig. 3. Time variation of the FCN phase. The FCN phase has two sudden trend changes in 1992 and 1998 . Two arrows plotted present epochs of geomagnetic jerks.

al., 1998). The CW is another free oscillation of polar motion of the Earth and their period is close to 435 mean solar days as viewed from the terrestrial frame (Lambeck, 1980). The largest phase disturbances, amounting $152^{\circ}$, happened in 1925. The broadest duration of the phase disturbance happened in 1953 and amounted to 15 years. Gibert et al. (1998) also found that the occurrence of the phase disturbances follow those of geomagnetic jerks with a delay not exceeding three years. A geomagnetic jerk is a rapid change in rate of the secular variation of geomagnetic fields. On the other hand, the theoretical model suggests that the instability of a layer at the top core and its downward propagation induce a step in the core-mantle torque strong enough to explain the phase disturbances in the $\mathrm{CW}$ (Bellanger et al., 2001).

The characteristic of time variation of the FCN phase is explained by the same mechanism on the phase disturbances in the $\mathrm{CW}$ though the numbers of disturbances are so different, two versus ten. One explanation is that at least one phase disturbance with a long duration occurred before the observation period which is responsible for the phase variation before 1992. Unfortunately accurate estimation of the parameters of each phase disturbances is not feasible due to the limited observation period. This is mainly because the impacts of the phase disturbances happened before 1992 and in 1998 remain beyond the observation period. Without those accurate estimations, it is difficult to perform accurate estimations of the parameters of the phase disturbance happened around 1992. On the other hands, the geomagnetic jerks occurred twice during the observation period, namely around 1992 and 1998 or 1999 (Huy et al., 1998; Huy et al., 2000; Mandea et al., 2000). These epochs coincide with the dates of the phase disturbances of the FCN as well as the CW. As a candidate of the phase disturbance before the observation period, the geomagnetic jerk occurred in 1979 (Huy et al., 1998) while the recent research suggests another might have occurred in 1983, too
(Wardinski et al., 2003).

\section{Conclusion}

We detected the phase disturbances of the FCN of the Earth directly from the analysis of the VLBI observation data for the period 1984-2003. During the period, the FCN had two significant phase disturbances in 1992 and 1998. These epochs coincide with the occurrence of the geomagnetic jerks. We have only two geomagnetic jerks during the limited observation period and its theoretical model has not been available yet. On the other hand, around ten phase disturbances in the Chandler wobble are reported to have occurred in the period 1870-1997. Those epochs are also close to those of the geomagnetic jerks (Gibert et al., 1998; Bellanger et al., 2002). A theoretical model for such phase disturbances in the $\mathrm{CW}$ is already provided by Bellanger $e t$ al. (2001). We regard that it is important to analyze the VLBI nutation data of a longer observation period and to construct a theoretical model for the FCN as well as the $\mathrm{CW}$. Since the origin of geomagnetic jerk is supposed to be inside of the Earth, it is an important issue to study the dynamics of the Earth's interior and the conductivity properties of the mantle. Therefore this phenomena could be a new diagnostic tool to investigate the Earth's interior through the VLBI data. Lastly we think it worth mentioning that a trend of the Earth's dynamic oblateness $J_{2}$ also changed suddenly in 1998 (or 1999) (Cox and Chao, 2002). Although its geophysical cause(s) are uncertain, they mention the geomagnetic jerk in 1998 as a potential geophysical cause. Windows application of the SPG method is available from the author TS.

Acknowledgments. Ferrandiz, J. M. and Heki, K. are deeply acknowledged for providing us with very constructive reviews.

\section{References}

Bellanger, E., J.-L. Le Mouel, M. Mandea, and S. Labrosse, Chandler wobble and geomagnetic jerks, Physics of the Earth and Planetary 
Interiors, 124, 95-103, 2001.

Bellanger, E., D. Gibert, and J.-L. Le Mouel, A geomagnetic triggering of Chandler wobble phase jumps?, Geophys. Res. Lett., 29, 28-1, 2002.

Cox, C. M. and B. F. Chao, Detection of a large-scale mass redistribution in the terrestrial system since 1998, Science, 297, 831-833, 2002.

Dehant, V., M. Feissel-Vernier, O. de Viron, C. Ma, M. Yseboodt, and C. Bizouard, Remaining error sources in the nutation at the submilliarc second level, J. Geophys. Res., 108, ETG 13-1, 2003.

Foster, G., Wavelets for period analysis of unevenly sampled time series, Astron. J., 112, 1709-1729, 1996.

Gabor, D., Theory of communication, 93, 429-457, 1946.

Getino, J. and J. M. Ferrandiz, Effects of dissipation and a liquid core on forced nutations in Hamiltonian theory, Geophys. J. I., 142, 703-715, 2000.

Gibert, D., M. Holschneider, and J.-L. Le Mouel, Wavelet analysis of the Chandler wobble, J. Geophys. Res., 103, 27069-27089, 1998.

Hinderer, J., J. P. Boy, P. Gegout, P. Defraigne, F. Roosbeek, and V. Dehant, Are the free core nutation parameters variable in time?, Earth Planet. Sci. Lett., 117, 37-49, 2000.

Huy, M. L., M. Alexandrescu, G. Hulot, and J.-L. Le Mouel, On the characteristics of successive geomagnetic jerks, Earth Planets Space, 50, 723-732, 1998.

Huy, M. L., M. Mandea, J.-L. Le Mouel, and A. Pais, Time evolution of the fluid flow at the top of the core. Geomagnetic jerks, Earth Planets Space, 52, 163-173, 2000.

Lambeck, K., The earth's variable rotation: Geophysical causes and consequences, Cambridge Monographs on Mechanics and Applied Mathematics, 1980.

Malkin, Z. and D. Terentev, Investigation of the Parameters of the Free Core Nutation from VLBI data, Communications of the IAA RAS, 149,
2003.

Mandea, M., E. Bellanger, and J.-L. Le Mouel, A geomagnetic jerk for the end of the 20th century?, Earth Planet. Sci. Lett., 183, 369-373, 2000.

Mathews, P. M., T. A. Herring, and B. A. Buffett, Modeling of nutation and precession: New nutation series for nonrigid Earth and insights into the Earth's interior, J. Geophys. Res., 107, ETG 3-1, 2002.

Roosbeek, F., P. Defraigne, M. Feissel, and V. Dehant, The free core nutation period stays between 431 and 434 sidereal days, Geophys. Res. Lett., 26, 131-134, 1999.

Sasao, T., S. Okubo, and M. Saito, A Simple Theory on Dynamical Effects of Stratified Fluid Core upon Nutational Motion of the Earth, Proceedings of IAU Symposium No. 78, 165, 1980.

Sato, T., Y. Tamura, T. Higashi, S. Takemoto, I. Nakagawa, N. Morimoto, Y. Fukuda, J. Segawa, and N. Seama, Resonance parameters of the free core nutation measured from three superconducting gravimeters in Japan, J. Geomag. Geoelect., 46, 571-586, 1994.

Shirai, T. and T. Fukushima, Construction of a new forced nutation theory of the nonrigid Earth, Astron. J., 121, 3270-3283, 2001a.

Shirai, T. and T. Fukushima, Detection of excitations of free core nutation of the Earth and their concurrence with huge earthquakes, Geophys. Res. Lett., 28, 3553-3557, 2001 b.

Toomre, A., On the '1nearly diurnal wobble' of the earth, Geophys. J., 38, 335-348, 1974.

Wardinski, I., M. Mandea, and R. Holme, The Origin of Geomagnetic Jerks, Revisited, AGU, Fall Meeting 2002, GP31D-0769, 2003.

T. Shirai (e-mail: toshimichi.shirai@gs.com), T. Fukushima, and Z. Malkin (e-mail: malkin@quasar.ipa.nw.ru) 